\title{
Pheomelanin-Based Coloration and the Ability to Cope with Variation in Food Supply and Parasitism
}

\author{
Romain Piault, ${ }^{1, *}$ Julien Gasparini, ${ }^{1,2}$ Pierre Bize, ${ }^{1,3}$ Susanne Jenni-Eiermann, ${ }^{4}$ and \\ Alexandre Roulin ${ }^{1}$
}

1. Department of Ecology and Evolution, Biophore, University of Lausanne, 1015 Lausanne, Switzerland; 2. Laboratoire Ecologie et Evolution, Université Pierre et Marie Curie, Centre National de la Recherche Scientifique Unité Mixte de Recherche 7103, 7 quai St. Bernard, 7625 Paris, France; 3. Division of Environmental and Evolutionary Biology, Institute of Biomedical and Life Sciences, Graham Kerr Building, Glasgow University, Glasgow G12 8QQ, United Kingdom; 4. Swiss Ornithological Institute, Sempach, Switzerland

Submitted February 2, 2009; Accepted May 1, 2009; Electronically published August 19, 2009

AвSTRACт: Although gene by environment interactions may play a key role in the maintenance of genetic polymorphisms, little is known about the ecological factors involved in these interactions. We investigated whether food supply and parasites can mediate covariation between the degree of adult pheomelanin-based coloration, a heritable trait, and offspring body mass in the tawny owl (Strix aluco). We swapped clutches between nests to allocate genotypes randomly among environments. Three weeks after hatching, we challenged the immune system of 80 unrelated nestlings with either a phytohemagglutinin (PHA) or a lipopolysaccharide, surrogates of alternative parasites, and then fed them ad lib. or food-restricted them during the following 6 days in the laboratory. Whatever the immune challenge, nestlings fed ad lib. converted food more efficiently into body mass when their biological mother was dark pheomelanic. In contrast, food-restricted nestlings challenged with PHA lost less body mass when their biological mother was pale pheomelanic. Nestling tawny owls born from differently melanic mothers thus show differing reaction norms relative to food availability and parasitism. This suggests that dark and pale pheomelanic owls reflect alternative adaptations to food availability and parasites, factors known to vary in space and time.

Keywords: genotype by environment interaction, pheomelanism, food availability, immune challenge, nestlings, tawny owl.

\section{Introduction}

Gene by environment interactions may be a potent force maintaining genetic variation in natural populations (Kassen 2002; Byers 2005; Chunco et al. 2007). Environmental conditions fluctuate over space and time in the wild, which, in turn, may create fluctuating selective pressures favoring different gene combinations or expressions at different times and spatial scales (i.e., selection for alternative

* Corresponding author; e-mail: romain.piault@unil.ch.

Am. Nat. 2009. Vol. 174, pp. 548-556. (C) 2009 by The University of Chicago. 0003-0147/2009/17404-51044\$15.00. All rights reserved.

DOI: $10.1086 / 605374$ reaction norms). Although the potential role of environmental heterogeneity in maintaining genetic variation has been the focus of theoretical works for more than half a century, empirical demonstrations of this phenomenon in natural populations remain limited to a few cases (reviewed in Hedrick 2006).

Melanin-based coloration is a suitable study system for understanding the influence of environmental heterogeneity on the amount of genetic variation in natural populations for at least two reasons. First, experimental studies often failed to demonstrate the condition dependence of melanic ornaments on nutrition and health status (e.g., McGraw 2008; but see Griffith et al. 2006), suggesting that the degree of melanin-based coloration is often under tight genetic control (Hearing and Tsukamoto 1991); thus, in many cases, melanin-based colors can be considered as phenotypic markers of alternative genotypes (Hoekstra 2006). Second, the degree of melanin-based coloration has been frequently reported to covary with life-history, morphological, physiological, and behavioral traits, with the sign and magnitude of these covariations fluctuating in space and over time (Roulin 2004). Hence, it suggests that the degree of melanin-based coloration reflects adaptations to different environmental conditions, a major prerequisite for gene by environment interactions to maintain genetic diversity (Kawecki and Ebert 2004). Eventually, a recent review has highlighted potential candidate genes pleiotropically affecting both the degree of individual melanization and other phenotypic traits, including energy homeostasis and anti-inflammatory immune reactions (Ducrest et al. 2008). This knowledge offers us the possibility to specifically target key environmental factors, such as food availability and parasites, which may modulate the performance of differently melanic individuals in the wild.

Using the tawny owl (Strix aluco) as a study system, we tested whether organisms that differed in the degree of melanin-based coloration respond differently (i.e., have 
different reaction norms) to variation in food availability and parasitism, a first step to understanding whether the degree of melanin-based coloration reflects adaptations to alternative environmental conditions. The tawny owl is a nocturnal raptor that shows continuous interindividual variation in the degree of pheomelanin-based plumage reddishness. In this species, the expression of plumage coloration shows high heritability $\left(h^{2}=0.76\right.$ to 0.93 ; Gasparini et al. 2009) and is not sensitive to sibling competition (i.e., brood size manipulation experiments; G. Emaresi, J. Gasparini, P. Bize, R. Piault, and A. Roulin, unpublished data). Variation in coloration is associated with indices of individual performance, such as immunity (Galeotti and Sacchi 2003; Gasparini et al. 2009) and offspring body mass growth (Roulin et al. 2003, 2004, 2008a). Dark pheomelanic females produced heavier offspring than pale pheomelanic ones in some years, while the reverse pattern was observed in other years (Roulin et al. 2003, 2004). Given that in mice pheomelanin-based coat coloration can be genetically related to regulation of energy homeostasis (Stutz et al. 2005) and proinflammatory responses to endotoxin challenges (Lipton et al. 1999), we hypothesized that differently colored tawny owls are adapted to different levels of food availability and parasite exposure, two factors known to affect nestling growth.

To test whether nestlings born from differently colored parents show different reaction norms relative to food availability and parasite exposure, we first swapped eggs between pairs of nests to allocate randomly the offspring produced by differently colored genotypes among environments. In this way, we could tease apart origin-related effects from influences of posthatching parental care on the nestlings' ability to grow. Note that prehatching maternal effects and genetic effects are still confounded with our design. Then, we compared body mass change of offspring produced by differently colored parents under four experimental settings that differed in the immune challenge (i.e., phytohemagglutinin [PHA] vs. lipopolysaccharide [LPS]), surrogates for alternative parasites, and food supply (i.e., food restricted vs. ad lib.). Three-week-old nestlings were challenged with the lectin PHA or an LPS and subsequently fed ad lib. or were food-restricted during the following 6 days. To gain insight into the physiological mechanisms underlying the association between body mass growth and coloration, we measured triglycerides (TG) and free fatty acids (FFA) as indicators of fat deposition (when food is abundant) and fat catabolism (during fasting), respectively (Jenni-Eiermann and Jenni 1997; Guglielmo et al. 2002). We predicted that, if differently colored tawny owls are adapted to different levels of food availability and parasite exposure, nestlings produced by dark pheomelanic parents would be favored (in terms of body mass change) in a given environment, while in an- other environment they would be outcompeted by nestlings produced by pale pheomelanic parents (i.e., crossover interaction).

\section{Methods}

The study was carried out in 2005 in a population of tawny owls located in western Switzerland, where 366 nest boxes have been installed in an area of $911 \mathrm{~km}^{2}$. The tawny owl reproduces from January to May. Females incubate eggs for $\sim 28$ days (Galeotti 2001). Nestlings grow rapidly depending on the availability of prey and fledge at 25-30 days of age (Galeotti 2001). Nestling body mass reaches a plateau around 23 days of age (R. Piault, unpublished data) and is associated with survival before dispersal (Overskaug et al. 1999). Nestling and adult tawny owls are infested by various blood parasites, with large spatial and annual variation in prevalence and intensity of infestation (e.g., Appleby et al. 1999; Galeotti and Sacchi 2003). In our population, we investigated blood parasitism in nestling and adult owls in 2004. We found low prevalence of hematozoan Haemoproteus, Leucocytozoon, and Trypanosoma spp. and blood flies Carnus haemapterus (A. Roulin and P. Galeotti, unpublished data). More work on annual variation in infestation rates is required to establish whether melanin-based coloration covaries with susceptibility to blood parasites.

\section{Measurement of Plumage Coloration}

Scores of plumage coloration were obtained following the methods described by Gasparini et al. (2009). Three feathers were collected per adult owl and then individually photographed with a digital camera (Dimage A200, Konika Minolta) fixed at a distance of $27 \mathrm{~cm}$ from the feather. Pictures were imported into the software Adobe Photoshop to measure hue, saturation, and brightness in 10 randomly chosen locations. We obtained a continuous coloration score by calculating mean hue, saturation, and brightness values over the 30 measurements per individual (10 measurements on each of three feathers). Because these three variables were highly intercorrelated (Pearson correlations: $|r| \geq 0.77, P<.0001$ for hue, saturation, and brightness), we extracted the first component (PC1) of a principal components analysis, which explained $87 \%$ of the total variance. $\mathrm{PC} 1$ provides a reliable measure of coloration that correlates with feather pheomelanin content and a color score obtained with a spectrophotometer, that is, hue, the wavelength for which feather reflectance is the highest (range: $300.5-662.5 \mathrm{~nm}$ on 230 adult owls captured in 2005; Gasparini et al. 2009). We gave preference to color measurements extracted from digital pictures rather than spectra because the use of pictures and Adobe Photoshop 
allows us to precisely target the colored parts of feathers, while this is not easy with a spectrophotometer probe. Tawny owl feathers show thin black lines interspaced with grayish to reddish colored parts, and because these latter are responsible for overall individual plumage coloration, we believe that the use of Adobe Photoshop is the best option to score plumage coloration in this species. Furthermore, tawny owls probably do not perceive UV colorations since no UV-sensitive photoreceptor class has been found using microspectrophotometry (Bowmaker and Martin 1978). Therefore, the measurement of feather reflectance in the ultraviolet A range is not strictly necessary even in the context of color signaling function. Low and high PC1 scores stand for dark and pale pheomelanic owls, respectively.

Although we preferentially use pictures to measure coloration, we also collected reflectance spectra using an Ocean Optics S2000 spectrophotometer (Dunedin, FL) and a Deuterium-Halogen 2000 light source (Mikropack, Ostfildern). Individual spectral hues were calculated following methods described by Montgomerie (2006). To show that our results are independent of the method of assessing coloration, we also report statistics obtained with reflectance spectra.

\section{Experimental Procedure}

In March 2005, we visited nest boxes to record clutch size (mean \pm SE: $4.2 \pm 0.1$ eggs, range: $2-6$ ), hatching date (April $5 \pm 1.2$ days; March 19-April 30), and brood size at hatching (3.6 \pm 0.1 nestlings; $2-5)$. We cross-fostered clutches between 40 pairs of nests on the basis of the criteria that within a pair clutches were laid on a similar date $(r=0.75, P<.0001)$. Clutches were randomly assigned with respect to plumage coloration of both biological and foster parents. Within pairs, plumage coloration of either biological parent did not correlate with plumage coloration of either foster parent (all $P>.10$ ). Females $(n=80)$ were captured while sitting on eggs or hatchlings, and males $(n=67)$ were captured when feeding $11.7 \pm 0.2$-day-old nestlings. Nestlings were banded with a numbered aluminium ring to recognize them individually on this latter occasion. Ten days later, we injected all siblings $(21.7 \pm 0.3$ days old $)$ in the wing web with the same antigen, that is, LPS ( $n=40$ nests, serotype 055:B5; Sigma, L2880; $20 \mu \mathrm{g}$ diluted in $0.02 \mathrm{~mL}$ ) or PHA ( $n=40$ nests, Sigma, L1668; $10 \mu$ g diluted in $0.02 \mathrm{~mL}$ ). PHA is derived from plant beans of Phaseolus vulgaris and specifically induces T-cell division in vitro (Mire-Sluis et al. 1987). LPS is obtained from degenerated cell walls of gram-negative bacteria, is a mitogen for B lymphocytes, and elicits a specific antibody response in vivo even in extremely small amounts (Skidmore et al. 1975). Both an- tigens induce acute phase responses, that is, metabolic adjustments that support immune responses (Klasing 1988). Because we had no a priori reason to think that major results would be detected with one particular immune challenge, we increased our chances to detect covariation between plumage coloration and immunity by using two contrasting immune challenges (PHA vs. LPS) rather than a single immune challenge and a control group (e.g., PHA vs. control). Furthermore, short breeding season and limited space in the laboratory did not allow us to keep more than 80 nestlings under standardized laboratory conditions; therefore, we could not create a third control group. However, the lack of control group is not a problem because our aim was to create two groups of nestlings growing in distinct environments (here mimicked by an injection of PHA vs. LPS) rather than to study the absolute cost of mounting a particular immune response.

After cutaneous immune responses to LPS and PHA were measured, we took one nestling per nest and brought it in the laboratory in the afternoon (day 0; mean nestling age: $22.2 \pm 0.3$ days). Nestlings were fed ad lib. until the next morning when food treatments started (day 1 ). We restricted our experiment to one individual per nest (i.e., 80 nestlings) because of time and space constraints in the laboratory. All nestlings brought to the laboratory were assumed unrelated (extrapair young are rare in the tawny owl; e.g., one extrapair young out of 137 nestlings in an adjacent Swiss population; Saladin et al. 2007). Nestlings were either fed ad lib. (i.e., they could eat as many laboratory mice as they wanted; on average $56.0 \pm 1.2 \mathrm{~g}$ day $^{-1}$; range: $38.7-75.5 \mathrm{~g}$ ) or food restricted (each nestling could eat two laboratory mice per day; on average $29.9 \pm 0.2 \mathrm{~g} \mathrm{day}^{-1}[53.4 \%$ of the ad lib. consumption]; range: $27.0-31.8 \mathrm{~g}$ ) for the remainder of their stay in the laboratory (i.e., 6 days). In natural conditions, nestlings usually consume between two and four small mammals per day (Galeotti 2001; main prey are wood mice Apodemus sp. [body mass range: $22-45 \mathrm{~g}$ ] and bank voles Clethrionomys glareolus [body mass range: $15-25 \mathrm{~g}]$ ). Hence, two laboratory mice are below what nestlings would eat in the wild under favorable environmental conditions. Details on the housing conditions of nestlings and setup of the food treatments, as well as the ethical note, can be found in the study by Piault et al. (2008). Nestling body mass, tarsus length, and wing length were measured to the nearest $0.1 \mathrm{~g}, 0.1 \mathrm{~mm}$, and $1 \mathrm{~mm}$, respectively, on day 1 between 0800 and 0900 hours. Nineteen (nine females and 10 males) of the 38 PHA-injected nestlings were assigned to the ad lib. treatment and the 19 (nine females and 10 males) others to the food-restricted treatment. Similarly, 21 LPS-injected nestlings were assigned to the ad lib. treatment (10 females and 11 males) and 21 to the food-restricted treatment (12 females and nine males). 
Table 1: Stepwise ANCOVAs on nestling body mass change in 6 days with immune challenges (PHA vs. LPS) as a factor and amount of food consumed and plumage coloration of the biological mother as a covariate (PC1 or spectral hue in parentheses)

\begin{tabular}{|c|c|c|c|c|c|c|c|c|}
\hline \multirow{3}{*}{$\begin{array}{l}\text { Source of } \\
\text { variation }\end{array}$} & \multicolumn{4}{|c|}{ Ad lib. } & \multicolumn{4}{|c|}{ Food restricted } \\
\hline & \multirow[b]{2}{*}{ Estimate $\pm \mathrm{SE}$} & \multicolumn{2}{|c|}{ Test statistic } & \multirow[b]{2}{*}{$P$} & \multirow[b]{2}{*}{ Estimate $\pm \mathrm{SE}$} & \multicolumn{2}{|c|}{ Test statistic } & \multirow[b]{2}{*}{$P$} \\
\hline & & $F$ & $\mathrm{df}$ & & & $F$ & $\mathrm{df}$ & \\
\hline $\mathrm{A}$ & $\begin{array}{c}.33 \pm .07 \\
(.29 \pm .07)\end{array}$ & $21.43(16.62)$ & 1,37 & $<.0001(.0002)$ & $\begin{array}{c}1.39 \pm .42 \\
(1.81 \pm .37)\end{array}$ & $11.18(20.80)$ & 1,35 & $.002(<.0001)$ \\
\hline I & $\begin{array}{c}1.61 \pm 3.17 \\
(4.07 \pm 3.25)\end{array}$ & $.26(1.56)$ & 1,36 & $.61(.22)$ & $\begin{array}{l}-5.76 \pm 2.46 \\
(-7.09 \pm 2.55)\end{array}$ & $5.48(7.72)$ & 1,35 & $.03(.009)$ \\
\hline Color & $\begin{array}{r}-5.26 \pm 1.90 \\
(.05 \pm .03)\end{array}$ & $7.65(4.27)$ & 1,37 & $.009(.046)$ & $\begin{array}{c}3.76 \pm 1.40 \\
(-.05 \pm .02)\end{array}$ & $7.17(7.20)$ & 1,35 & $.01(.01)$ \\
\hline $\mathrm{I} \times$ color & $\begin{array}{r}-.88 \pm 1.91 \\
(.04 \pm .03)\end{array}$ & $.21(2.25)$ & 1,35 & $.65(.14)$ & $\begin{array}{r}-3.09 \pm 1.45 \\
(.04 \pm .02)\end{array}$ & $4.52(3.50)$ & 1,35 & $.04(.07)$ \\
\hline
\end{tabular}

Note: PHA, phytohemagglutinin; LPS, lipopolysaccharide; A, amount of food consumed; I, immune challenges. Separate tests were performed for each food treatment. All nonsignificant terms were removed from the final analyses, explaining why degrees of freedom vary between factors. Estimates \pm SE are the coefficients of the linear model found by least squares.

Nestlings were returned to their original nest on day 7 after body mass, tarsus, and wing lengths had been measured a second time in the morning (at 0800 hours). During the 6 days of food treatment, nestlings fed ad lib. gained body mass and size (mean \pm SE; body mass: $15.3 \pm 3.8$ g; wing length: $34.5 \pm 0.6 \mathrm{~mm}$; tarsus length: $3.8 \pm 0.3$ $\mathrm{mm}$ ), while food-restricted nestlings lost body mass $(-33.3 \pm 3.3 \mathrm{~g})$ and gained $32.6 \pm 0.6 \mathrm{~mm}$ of wing length and $2.7 \pm 0.2 \mathrm{~mm}$ of tarsus length. Thus, our food treatments effectively created two groups of nestlings differing in nutritional status (one-way ANOVAs with body mass change, wing growth, or tarsus growth as the dependent variable and food treatment as a factor; all $P \leq .02$ ).

Nestling body mass, tarsus length, wing length, and nestling rank in the age hierarchy in the brood of rearing did not differ between the four treatments at the start of the experiment (two-way ANOVA with food treatment and immune challenge as fixed factors plus their interaction; all $P>.13$ ). Biological and foster parents of the nestlings allocated into the four groups did not differ in coloration (two-way ANOVA with food treatment and immune challenge as fixed factors plus their interactions; all $P>.24$ ). There was no significant correlation between the coloration of the biological mother and the coloration of the foster mother in the ad lib. group $(r=0.001, n=40$, $P=1$ ). Within the food-restricted treatment, plumage coloration of the biological mother did not correlate significantly with plumage coloration of the foster mother either in the PHA-injected group $(r=-0.16, n=19$, $P=.51)$ or in the LPS-injected group $(r=-0.35, n=$ $21, P=.12$ ). In each of the four treatment groups, plumage coloration of the biological mother did not correlate with plumage coloration of the foster male (all $P>.18$ ). In each of the four experimental groups, mother plumage coloration did not covary with mother body mass measured during incubation (ANCOVA with mother body mass as dependent variable, group as a factor, coloration as a covariate plus interaction; all $P>.38$ ). Plumage coloration of the foster parents is not considered hereafter, since this was not the focus of the study. Since the body mass change was similar between male and female nestlings within each feeding treatment group (ANCOVA models presented in table 1 with sex entered as a factor; $P>.72$ ), we do not consider nestling sex any further in the statistical analyses.

\section{Measurement of Triglycerides (TG) and Free Fatty Acids (FFA)}

The day owlets were returned to their nest (day 7), we collected a blood sample from their brachial vein to determine nestling blood concentrations of TG and FFA and nestling sex. Blood samples were taken in the field between 0840 and 1950 hours (mean \pm SD; $1156 \pm 0251$ hours) once nestlings arrived to their nests. In the statistical analyses, we entered the time of day at which blood samples were collected (referred to hereafter as time of blood collection). Nestlings were not fed during their travel back to their original nest, and thus time of their last meal is unknown. After centrifugation, red blood cells and plasma were stored at $-20^{\circ} \mathrm{C}$ until sex determination and metabolite assays. Metabolites were determined using standard test combinations as modified for handling small amounts of plasma (3-10 $\mu \mathrm{L}$ per determination): enzymatic colorimetric tests for FFA (Wako NEFA C test kit) and TG, including free glycerol (Wako GPO-DAOS method). Within plates, blood concentration values for the same sample were highly repeatable for both TG and FFA $\left(r_{\mathrm{TG}}=0.87, P<.0001 ; r_{\mathrm{FFA}}=0.88, P<.0001\right)$. Nestlings were sexed following the molecular methods described by Py et al. (2006). 


\section{Statistics}

Statistical analysis was performed using JMP IN 7.0.0. To test whether coloration of both biological parents explained variation in nestling body mass change over 6 days in the laboratory in relation to the food treatment and the type of immune challenge, we performed an ANCOVA with nestling body mass change (the difference between body masses measured at days 7 and 1) as the dependent variable. We entered food treatments and immune challenges as factors, plumage coloration of both biological parents as covariates, plus all possible three-way interactions. Analyses were conducted twice, using either the Photoshop-derived PC1 color score or individual hues obtained with a spectrophotometer as plumage coloration scores. Outputs of both types of models are presented in tables to show that they led to qualitatively similar results. However, interpolations and graphs are drawn from models including PC1 because we believe it better measures variation in the degree of pheomelanism. We ran full factorial models and then dropped nonsignificant terms (starting with nonsignificant interactions) in a stepwise manner in order to produce minimum adequate final models. Throughout the article, means \pm SEs are quoted unless otherwise stated; statistical tests are two tailed, and significance level is set to 0.05 . In all models, residuals were normally distributed, and variances were homogeneous between treatments.

\section{Results}

After stepwise backward simplification of the initial statistical model including plumage coloration of both biological parents, only the coloration of the mother was retained in two-way interactions with food treatment and immune challenge, respectively (table 2 ).

Within the ad lib. treatment, nestlings gained more mass when their biological mother was dark pheomelanic, irrespective of the immune challenge, and after controlling for total food consumption (table 1 ; fig. $1 A$ ). Inclusion of nestling total food consumption in the model is valid because appetite was not affected by the immune challenges (mass of mice consumed during 6 days by LPS- vs. PHAinjected nestlings from the ad lib. treatment: $343.5 \pm$ 8.8 vs. $327.7 \pm 11.4 \mathrm{~g}$; Student's $t$-test, $t=-1.11$, $\mathrm{df}=38, P=.27)$. Furthermore, nestling appetite did not correlate significantly with plumage coloration of the biological mother (Spearman correlation, $R=0.20, n=$ $40, P=.22$ ). Interpolations from the model indicate that nestlings produced by the reddest mothers would convert $400 \mathrm{~g}$ of mice into $52.5 \pm 8.8 \mathrm{~g}$ of body mass, while those of the least reddish mothers would convert the same amount of mice into $20.9 \pm 6.9 \mathrm{~g}$.

Using similar statistical analyses on TG plasma concentrations measured in nestlings fed ad lib., we found a significant interaction between coloration of the biological mother and food consumption during the last 24 hours preceding blood sampling (i.e., day 6; multiple regression with nestling TG concentration as the dependent variable; time of blood collection, food consumption, and coloration of the biological mother [PC1] as covariates plus their interaction; interaction coloration of the biological mother $\times$ food consumption: $\left.F_{1,35}=4.51, P=.04\right)$. By designating mothers to two classes of coloration (dark and pale pheomelanic) on the basis of median coloration, TG concentration was positively correlated with the amount of food consumed the previous day in nestlings born from dark pheomelanic mothers after controlling for the variable "time of blood collection" $\left(F_{1,16}=11.19, P=.004\right)$ but not in nestlings produced by pale pheomelanic mothers $\left(F_{1,17}=0.65, P=.43\right)$. In a similar multiple regression analysis, we found no significant relationship between FFA blood concentration and plumage coloration of the bio-

Table 2: Stepwise ANCOVA on nestling body mass change over 6 days (dependent variable) with food treatments (ad lib. vs. food restricted) and immune challenges (PHA vs. LPS) as two factors and plumage coloration of the biological mother as a covariate (PC1 or spectral hue in parentheses)

\begin{tabular}{lcccc}
\hline \multirow{2}{*}{$\begin{array}{l}\text { Source of } \\
\text { variation }\end{array}$} & Estimate $\pm \mathrm{SE}$ & \multicolumn{2}{c}{ Test statistic } & \\
\cline { 3 - 5 } & $23.78 \pm 2.36(23.88 \pm 2.37)$ & $101.34(101.08)$ & 1,73 & $<.0001(<.0001)$ \\
$\mathrm{F}$ & $-.56 \pm 2.36(-.44 \pm 2.43)$ & $.06(.03)$ & 1,73 & $.81(.86)$ \\
$\mathrm{I}$ & $.83 \pm 1.38(-.002 \pm .019)$ & $.36(.01)$ & 1,73 & $.55(.92)$ \\
Color & $4.68 \pm 2.36(6.20 \pm 2.42)$ & $3.92(6.54)$ & 1,73 & $.05(.01)$ \\
$\mathrm{F} \times \mathrm{I}$ & $-3.57 \pm 1.37(.05 \pm .02)$ & $6.79(6.14)$ & 1,73 & $.01(.02)$ \\
$\mathrm{F} \times$ color & $-3.27 \pm 1.37(.04 \pm .02)$ & $5.68(4.56)$ & 1,73 & $.02(.04)$ \\
$\mathrm{I} \times$ color & $1.46 \pm 1.38(.001 \pm .019)$ & $1.12(.003)$ & 1,72 & $.29(.96)$ \\
$\mathrm{F} \times \mathrm{I} \times$ color &
\end{tabular}

Note: PHA, phytohemagglutinin; LPS, lipopolysaccharide; F, food treatments; I, immune challenges. We removed the nonsignificant $(P=.29$ and .96 for $\mathrm{PC1}$ and spectral hue, respectively) triple interaction coloration of biological mother $\times$ food treatments $\times$ immune challenges from the final model, explaining why degrees of freedom vary between factors. Estimates \pm SE are the coefficients of the linear model found by least squares. 
A

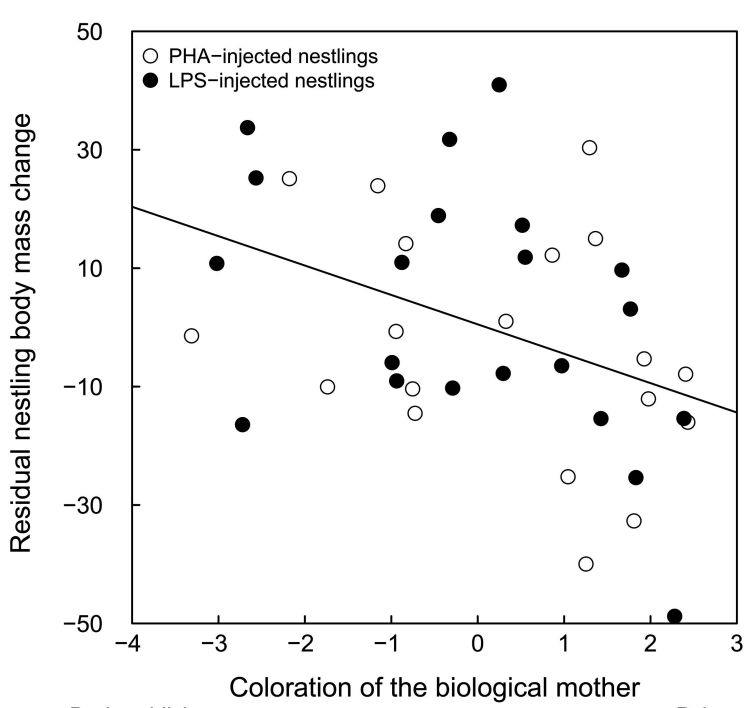

Dark reddish

B

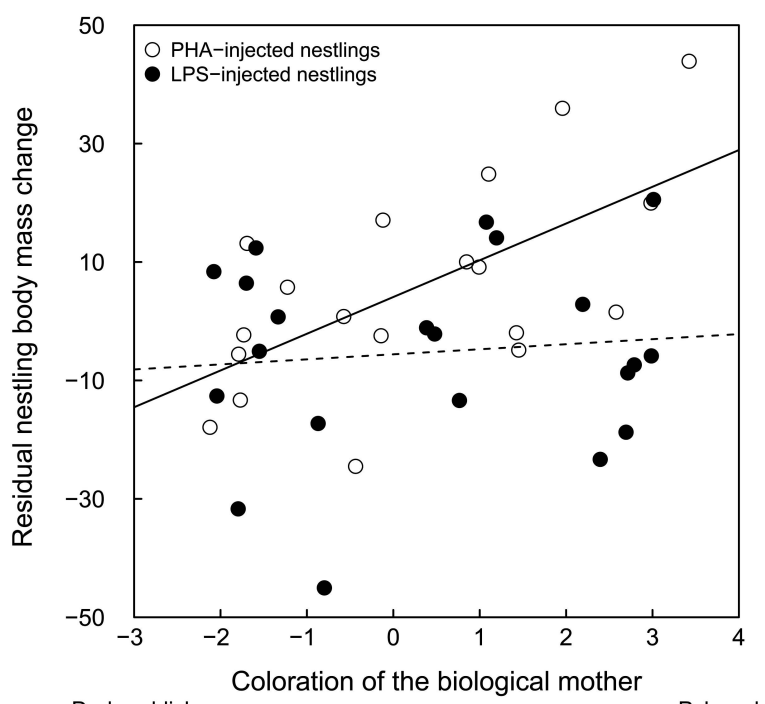

Dark reddish

Pale reddish

Figure 1: Relationship between plumage coloration of biological mother (PC1) and residual body mass change of nestlings fed ad lib. $(A)$ and nestlings fed under a restricted food regime $(B)$. Residual nestling body mass change was extracted from linear regressions of body mass change during 6 days on amount of food consumed during the same time period for each food treatment. Open and filled circles represent nestlings injected with phytohemagglutinin (PHA) and lipopolysaccharide (LPS), respectively. Within the food-restricted treatment, the relationship is significant for PHA-challenged nestlings (Pearson correlation; with PC1: $r=0.63, n=19, P=.004$; with spectral hue: $r=-0.62, n=19$, $P=.005$ ) but not for LPS-challenged ones (with PC1: $r=0.10, n=$ 21, $P=.67$; with spectral hue: $r=-0.14, n=21, P=.55)$. Regression lines are drawn. logical mother neither alone nor in the interaction with food consumption (all $P>.17$ ).

In the food-restricted treatment, and only when nestlings were challenged with PHA but not with LPS, nestlings lost significantly more body mass when their mother was darker pheomelanic with or without including nestling food consumption in the model (significant interaction between biological mother coloration and immune challenge in table 1; fig. 1B). Interpolations from the PHAinjected group show that for $180 \mathrm{~g}$ of mice eaten, nestlings produced by the reddest mothers lost $48.5 \pm 7.7 \mathrm{~g}$ of body mass, while those of the least reddish mothers lost only $8.8 \pm 6.5$ g. In the food-restricted and PHA-injected group, nestlings produced by paler pheomelanic females tended to have higher plasma concentrations of TG $\left(F_{1,14}=4.22, P=.06\right)$, after controlling for time of blood collection and food consumption in the previous day, while the relationship in the food-restricted and LPS-challenged group was far from being significant $\left(F_{1,17}=0.17\right.$, $P=.69)$. There was no significant relationship between FFA concentration in nestlings and the plumage coloration of their biological mother in both food treatments (all $P>.42$ ).

\section{Discussion}

We found that, independent of the immune challenge, nestlings born from dark pheomelanic mothers converted food more efficiently into body mass when fed ad lib. than offspring born from paler pheomelanic mothers. In contrast, nestlings born from dark pheomelanic mothers suffered greater body mass losses than their counterparts born from paler pheomelanic females when food restricted and challenged with PHA. The relationships between nestling body mass change and mother coloration within each experimental setting were linear, and thus nestlings born from mothers of intermediate levels of pheomelanism had intermediate variation in body mass as compared with nestlings produced by mothers of extreme phenotypes (i.e., dark and pale pheomelanic ones). Hence, significant pheomelanin-based color by environment interactions indicate that nestling tawny owls from differently colored mothers have alternative reaction norms for body mass in response to food availability and parasitism. It suggests that offspring produced by dark pheomelanic females are favored under favorable environmental conditions while they are outcompeted by offspring of less pheomelanic mothers under harsh environmental conditions (for a similar conclusion, see Roulin et al. 2008a).

Alternatively, relationships between plumage coloration of the biological mother and nestling body mass changes may be mediated by a condition-dependent effect. Under this hypothesis, biological mothers in good condition de- 
posit more pheomelanin into their feathers and thus develop a darker reddish-brown plumage than mothers in bad condition. Although we cannot discard this interpretation, the scope for a large condition-dependent effect on plumage coloration in the tawny owl that would change dark pheomelanic individuals exposed to harsh conditions during moult into paler pheomelanic ones is, however, limited. As stated in the "Introduction," tawny owl plumage coloration shows a heritability of $0.76\left(h^{2}=0.93\right.$ without an outlier possibly as a result of an extrapair paternity; Gasparini et al. 2009), suggesting that variation in plumage coloration is mainly driven by additive genetic variance in this species. Note also that plumage coloration remains stable throughout life, indicating a quite strong canalization of this phenotype. Furthermore, although the remaining unexplained variation in plumage coloration could be due to an unmeasured environmental effect, it also most certainly includes measurement errors and possibly nongenetic additive effects (Lynch and Walsh 1998; Kruuk 2004). For these reasons, we believe that if an environmental component of color variation exists in the tawny owl, it is probably of small extent, and our results mainly reflect a genotype by environment interaction rather than a condition-dependent effect.

Alternative reaction norms for body mass of offspring of tawny owls can arise if body mass gain under favorable conditions is traded off against body mass loss under harsh conditions (i.e., growth vs. maintenance trade-off). Interestingly, dark and pale pheomelanic birds are at the opposite ends of the trade-off in body mass regulation, with dark individuals maximizing body mass gain when environmental conditions are favorable and pale individuals better resisting poor environmental conditions. It is common consent that trade-offs in fitness components across environments are underlain by costs of adaptations and that they themselves can arise from two sources (Kassen 2002). The first is antagonistic pleiotropy, where genes favorable in one environment are deleterious in other environments. In line with this hypothesis, hormones of the melanocortin system are known to simultaneously determine the degree of pheomelanin-based coloration and confer advantages or disadvantages depending on the quality of the environment. For instance, in laboratory mice, ectopic expression of the agouti signalling protein (ASIP) is associated with yellow pheomelanic coat color, increased food conversion efficiency and fat storage under ad lib. feeding regime (Stutz et al. 2005), and increased sensitivity to stress, which may lead to adverse effects on body mass. Ectopic expression of ASIP was found to enhance glucocorticoids release and feeding inhibition following restraint and isolation, respectively (De Souza et al. 2000; Bazhan et al. 2004). Furthermore, glucocorticoids increase energy expenditure, accelerate net protein breakdown, and in- crease gluconeogenesis and hyperglycemia (Tataranni et al. 1996). In conjunction with the observation that food restriction can induce marked elevation of glucocorticoids (Sabatino et al. 1991), this suggests that mice with ectopic expression of agouti would suffer from greater body mass losses under food scarcity than their wild-type counterparts. Altogether, this raises the possibility that in the tawny owl, variation in the pattern of expression of ASIP or a similar endogenous antagonist of melanocortin receptors to ASIP (such as agouti-related protein; Stutz et al. 2005) is at least partly responsible for the degree of plumage pheomelanism a female displays and the way energy balance is regulated in her offspring. In support for this view, we found, in the ad lib. group, a positive relationship between plasma concentration of TG and food consumption in nestlings produced by dark pheomelanic mothers but not in those produced by pale females. As observed in mice with ectopic expression of ASIP (Stutz et al. 2005), it suggests that the former nestlings gain more body mass than the latter through increased fat storages per unit of food ingested. Furthermore, we found that in the food-restricted and PHA-challenged group, nestlings produced by pale pheomelanic females tended to have higher plasma concentrations of TG compared with nestlings produced by dark pheomelanic females. This suggests that nestlings of pale pheomelanic females limit use of TG derived from food through a lower metabolic rate allowing sustained TG blood level.

The second source of costs of adaptations is the accumulation of mutations that are neutral in the environment of selection but deleterious in other environments (Kassen 2002). Although we cannot reject this hypothesis, our results do not support it. Indeed, if, for instance, dark pheomelanic females perform relatively worse in harsh environmental conditions because of the accumulation of mutations that are deleterious in stressful but not in mild environments, we expect all females to perform equally well under mild conditions independent of their degree of pheomelanism (i.e., no relationship between plumage coloration of the biological mother and nestling body mass change in the ad lib. treatment). Our study reveals that this is not the case, since nestling body mass gain linearly increased with the degree of pheomelanism under an ad lib. regime.

Independent of the genetics underlying our pattern of gene by environment interaction, our results suggest that environmental heterogeneity in food abundance and parasite exposure may help maintain diversity in the degree of pheomelanin-based coloration. Both environmental factors show spatial and temporal fluctuations. For instance, wood mice (Apodemus sp.) and bank voles (Clethrionomys glareolus), the main preys of tawny owls in our population (Roulin et al. 2008b), show 3-4-year cycles in their abun- 
dance. In the same way, prevalence of hematozoan parasites in nestling tawny owls was found to dramatically vary between years and broods in an English population (Appleby et al. 1999). To clearly establish a role for environmental heterogeneity in the maintenance of diversity in the degree of pheomelanism, two points need to be clarified. First, all nestlings that were food restricted and challenged with LPS did equally badly, regardless of the coloration of their biological mother. The outcome of nestling food restriction and LPS challenging suggests that when food is scarce and nestlings are infected with pathogens that activate energetically costly immune responses or particular immune branches, all mother phenotypes are counterselected. Whether such a situation frequently occurs in natural conditions is therefore crucial to determine whether parasites contribute to the maintenance of color diversity in the tawny owl. Second, it is still unknown in the tawny owl whether population growth rate (i.e., fitness) is weakly or strongly sensitive to changes in nestling growth. This question needs to be addressed to determine the extent to which environmental factors affecting nestlings influence the coexistence of diverse pheomelaninbased phenotypes in mothers.

In conclusion, we provide experimental evidence that tawny owls born from differently pheomelanic mothers show differing reaction norms relative to food availability and parasitism. In particular, variation in the degree of maternal pheomelanism seems aligned along a trade-off between offspring ability to gain body mass under high food abundance and offspring resistance to body mass losses under short periods of food scarcity. A review of the literature points to candidate genes potentially explaining the existence of such a trade-off: hormones of the melanocortin system are well known to pleiotropically affect the degree of pheomelanin-based coloration and regulation of energy homeostasis in vertebrate models such as mice. Additional studies are needed to challenge this hypothesis in the tawny owl and to test to what extent nestling body mass growth influences fitness in this species. This latter information is important to determine whether the present gene by environment interaction on nestling body mass change may help maintain the diversity in the degree of pheomelanin-based coloration in tawny owls.

\section{Acknowledgments}

We wish to thank K. Hine and anonymous reviewers for comments on earlier versions of the manuscript. This study was supported by the Swiss National Science Foundation (grants PPOOA-102913 to A.R. and PPOOA-109009 to P.B.) and the Roche Research Foundation (grant Mkl/stm 14-2008 to R.P.). The experiment was approved by the veterinary services of Canton de Vaud (licence 1508), and birds were ringed under the legal authorization of the Swiss Agency for the Environment, Forests, and Landscape.

\section{Literature Cited}

Appleby, B. M., M. A. Anwar, and S. J. Petty. 1999. Short-term and long-term effects of food supply on parasite burdens in tawny owls, Strix aluco. Functional Ecology 13:315-321.

Bazhan, N. M., A. Y. Shevchenko, N. R. Karkaeva, T. V. Yakovleva, and E. N. Makarova. 2004. Agouti yellow mutation increases adrenal response to ACTH in mice. European Journal of Endocrinology 151:265-270.

Bowmaker, J. K., and G. R. Martin. 1978. Visual pigments and colorvision in a nocturnal bird, Strix aluco (tawny owl). Vision Research 18:1125-1130.

Byers, D. L. 2005. Evolution in heterogeneous environments and the potential of maintenance of genetic variation in traits of adaptive significance. Genetica 123:107-124.

Chunco, A. J., J. S. McKinnon, and M. R. Servedio. 2007. Microhabitat variation and sexual selection can maintain male color polymorphisms. Evolution 61:2504-2515.

De Souza, J., A. A. Butler, and R. D. Cone. 2000. Disproportionate inhibition of feeding in $\mathrm{A}(\mathrm{y})$ mice by certain stressors: a cautionary note. Neuroendocrinology 72:126-132.

Ducrest, A. L., L. Keller, and A. Roulin. 2008. Pleiotropy in the melanocortin system, coloration and behavioural syndromes. Trends in Ecology \& Evolution 23:502-510.

Galeotti, P. 2001. Strix aluco tawny owl. BWP Update 3:43-77.

Galeotti, P., and R. Sacchi. 2003. Differential parasitaemia in the tawny owl (Strix aluco): effects of colour morphs and habitat. Journal of Zoology (London) 261:91-99.

Gasparini, J., P. Bize, R. Piault, K. Wakamatsu, J. D. Blount, A.-L. Ducrest, and A. Roulin. 2009. Strength and cost of mounting an immune response are associated with a heritable melanin-based color trait in female tawny owls. Journal of Animal Ecology 78: 608-616.

Griffith, S. C., T. H. Parker, and V. A. Olson. 2006. Melanin- versus carotenoid-based sexual signals: is the difference so black and red? Animal Behaviour 71:749-763.

Guglielmo, C. G., P. D. O'Hara, and T. D. Williams. 2002. Extrinsic and intrinsic sources of variation in plasma lipid metabolites of free-living western sandpipers (Calidris mauri). Auk 119:437-445.

Hearing, V. J., and K. Tsukamoto. 1991. Enzymatic control of pigmentation in mammals. FASEB Journal 5:2902-2909.

Hedrick, P. W. 2006. Genetic polymorphism in heterogeneous environments: the age of genomics. Annual Review of Ecology, Evolution, and Systematics 37:67-93.

Hoekstra, H. E. 2006. Genetics, development and evolution of adaptive pigmentation in vertebrates. Heredity 97:222-234.

Jenni-Eiermann, S., and L. Jenni. 1997. Diurnal variation of metabolic responses to short-term fasting in passerine birds during the postbreeding, molting and migratory period. Condor 99:113-122.

Kassen, R. 2002. The experimental evolution of specialists, generalists, and the maintenance of diversity. Journal of Evolutionary Biology 15:173-190.

Kawecki, T. J., and D. Ebert. 2004. Conceptual issues in local adaptation. American Naturalist 7:1225-1241.

Klasing, K. C. 1988. Nutritional aspects of leukocytic cytokines. Journal of Nutrition 118:1436-1446.

Kruuk, L. E. B. 2004. Estimating genetic parameters in natural pop- 
ulations using the "animal model." Philosophical Transactions of the Royal Society B: Biological Sciences 359:873-890.

Lipton, J. M., H. Zhao, T. Ichiyama, G. S. Barsh, and A. Catania. 1999. Mechanisms of antiinflammatory action of alpha-MSH peptides: in vivo and in vitro evidence. Annals of the New York Academy of Sciences 885:173-182.

Lynch, M., and B. Walsh. 1998. Genetics and analysis of quantitative traits. Sinauer, Sunderland, MA.

McGraw, K. J. 2008. An update on the honesty of melanin-based color signals in birds. Pigment Cell and Melanoma Research 21: 133-138.

Mire-Sluis, A. R., R. G. Wickremasinghe, A. V. Hoffbrand, A. M. Timms, and G. E. Francis. 1987. Human T lymphocytes stimulated by phytohaemagglutinin undergo a single round of cell division without a requirement for interleukin-2 or accessory cells. Immunology 60:7-12.

Montgomerie, R. 2006. Analyzing colors. Pages 90-147 in G. E. Hill and K. J. McGraw, eds. Bird coloration. Vol. 1. Mechanisms and measurements. Harvard University Press, Cambridge, MA.

Overskaug, K., J. P. Bolstad, P. Sunde, and I. J. Oien. 1999. Fledgling behavior and survival in northern tawny owls. Condor 101:169174.

Piault, R., J. Gasparini, P. Bize, M. Paulet, K. J. McGraw, and A. Roulin. 2008. Experimental support for the make-up hypothesis in nestling tawny owls (Strix aluco). Behavioral Ecology 19:703709.

Py, I., A. L. Ducrest, N. Duvoisin, L. Fumagalli, and A. Roulin. 2006. Ultraviolet reflectance in a melanin-based plumage trait is heritable. Evolutionary Ecology Research 8:483-491.

Roulin, A. 2004. The evolution, maintenance and adaptive function of genetic colour polymorphism in birds. Biological Reviews 79: $815-848$.

Roulin, A., B. Ducret, P. A. Ravussin, and R. Altwegg. 2003. Female colour polymorphism covaries with reproductive strategies in the tawny owl Strix aluco. Journal of Avian Biology 34:393-401.
Roulin, A., P. Bize, P. A. Ravussin, and L. Broch. 2004. Genetic and environmental effects on the covariation between colour polymorphism and a life-history trait. Evolutionary Ecology Research 6:1253-1260.

Roulin, A., J. Gasparini, P. Bize, M. Ritschard, and H. Richner. 2008a. Melanin-based colorations signal strategies to cope with poor and rich environments. Behavioral Ecology and Sociobiology 62:507519.

Roulin, A., B. Ducret, P. Bize, R. Piault, and P.-A. Ravussin. $2008 b$. Régime alimentaire de la chouette hulotte Strix aluco en Suisse romande de 1986 à 2007. Nos Oiseaux 55:149-156.

Sabatino, F., E. J. Masoro, C. A. McMahan, and R. W. Kuhn. 1991. Assessment of the role of the glucocorticoid system in aging processes and in the action of food restriction. Journals of Gerontology 46:B171-B179.

Saladin, V., M. Ritschard, A. Roulin, P. Bize, and H. Richner. 2007. Analysis of genetic parentage in the tawny owl (Strix aluco) reveals extra-pair paternity is low. Journal of Ornithology 148:113-116.

Skidmore, B. J., J. M. Chiller, D. C. Morrison, and W. O. Weigle. 1975. Immunological properties of bacterial lipopolysaccharide (Lps): correlation between mitogenic, adjuvant, and immunogenic activities. Journal of Immunology 114:770-775.

Stutz, A. M., C. D. Morrison, and G. Argyropoulos. 2005. The agoutirelated protein and its role in energy homeostasis. Peptides 26: 1771-1781.

Tataranni, P. A., D. E. Larson, S. Snitker, J. B. Young, J. P. Flatt, and E. Ravussin. 1996. Effects of glucocorticoids on energy metabolism and food intake in humans. American Journal of Physiology 34: E317-E325.

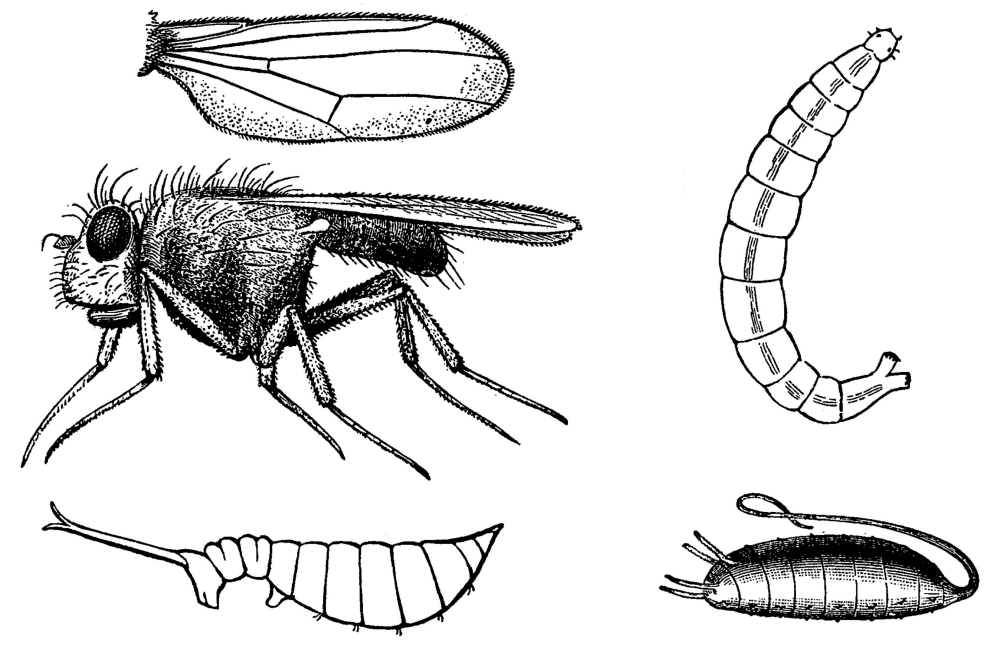

Left, "brine-inhabiting Ephydra." Top right, "insect we have found late in April at low-water mark, in Casco Bay, Maine, and like the Chironomus, living in the green seaweed." Lower right, "pupa of Eristalis, or Rat-tailed fly, found at the Equality Salt-works, Gallatin County, Illinois." From "Insects Living in the Sea" by A. S. Packard Jr. (American Naturalist, 1868, 2:277-279) 